\title{
Perfil socio-demográfico del donante de sangre del Hospital Nacional Cayetano Heredia- Lima: Una comparación entre 1988 y 1994*.
}

\author{
DE LA CRUZ DEL SOLAR Rafael, PINO GODOY Pavel, VIDAL ESCUDERO Julio** y RODRIGUEZ SALAZAR
} Isaías***

\section{SUMMARY}

All the people who make a blood donation is asked about if the blood could be used for transfusion or just for research. In the 80's, there was many information diffusion about this topic, but we don't know if this campaignes worked to avoid non elegible blood donors make a blood donation. Objective: To compare socio demographic characteristics between 1988 and 1994 blood donors to evaluate this. Material and methods: We made a comparative-retrospective study between historical groups. We selected 627 and 606 blood donors in 1988 and 1994 respectively. Results: $98 \%$ of blood donors in 1988 and $93.2 \%$ in 1994, hadn't any of the diseases asked, $2.5 \%$ in 1988 mantained homosexual intercourses sometime, in contrast $0 \%$ in $1994(\mathbf{p}<0.01)$ and $8.3 \%$ in 1988 mantained sexual intercourses with prostitutes or unknowed people in the last 6 months, in 1994 the incidence was 6.4\%. Conclusion: We found less high risk factors between 1994 blood donors than 1988, so the potential transmision of diseases in window period was minor. The selection of blood donors, rational use of transfusion and serologic screening are the main ways to make a good hemotherapy. ( Rev Med Hered 2000; 11:42-47).

KEY WORDS: Blood donors, transfusion, hemotherapy.

\section{RESUMEN}

Todas las personas que efectúan una donación de sangre son entrevistadas indirectamente, a través de una serie de preguntas en forma confidencial, si su donación puede ser usada para transfusiones o solo para estudio a fin de excluir aquellas de alto riesgo para la transmisión de enfermedades. En la década del 80 a raíz de la emergente epidemia de SIDA, se llevan a cabo campañas de difusión a través de los medios de comunicación mas usados (radio, televisión y periódicos) para que aquellas personas con antecedentes de riesgo se autoexcluyan del procedimiento. Objetivo: Comparar las características sociodemográficas entre donantes de los años 1988 y 1994 con el fin de buscar variaciones que pudieran sugerir su efectividad. Material y métodos: Se elaboró un estudio retrospectivo comparativo entre grupos históricos, seleccionándose del total de donantes por cada año, muestras aleatorias de 627

\footnotetext{
* Trabajo realizado en el Banco de Sangre del Hospital Cayetano Heredia Lima- Perú.

** Hematólogo Clínico. Jefe del Banco de Sangre, Hospital Nacional Cayetano Heredia.

*** Patólogo Clínico. Jefe del Dpto. Laboratorio Clínico, Hospital Nacional Cayetano Heredia.
} 
y 606 donantes en 1988 y 1994 respectivamente, evaluándose las respuestas a las preguntas de una encuesta que se aplica después de efectuar la donación. Resultados: El 98\% de donantes en 1988 y $93.2 \%$ en 1994 (p<0.0001) manifestó no haber sufrido ninguna de las enfermedades consultadas. El 2.5\% de donantes en 1988 manifestó haber experimentado relaciones homosexuales alguna vez en su vida, lo cual contrasta con el 0\% en 1994 ( $\mathrm{p}<0.01$ ) y el 8.3\% de donantes en 1988 reconoció haber tenido relaciones sexuales con prostitutas o desconocidos en los últimos 6 meses, mientras que en 1994 constituyó el 6.4\%. Conclusión: Hemos encontrado que el porcentaje de antecedentes de riesgo en donantes de sangre de 1994 fue menor que en 1988, con lo cual podría haberse reducido la transmisión de enfermedades infecciosas en el periodo ventana, ya que en cuanto a la serología no hubo diferencia significativa. La adecuada selección de donantes, el uso racional de las transfusiones y el screening serológico constituyen los principales medios para asegurar la inocuidad de la hemoterapia. ( Rev Med Hered 2000; 11:42-47).

PALABRAS CLAVE: Donante de sangre, transfusión, hemoterapia.

\section{INTRODOUCCION}

Todas las personas que acuden a efectuar una donación de sangre son entrevistadas indirectamente a través de una serie de preguntas en forma confidencial para determinar si su donación puede ser usada para transfusiones o solo para estudio. Este procedimiento se efectúa de manera rutinaria en todos los bancos de sangre del mundo, de modo que la sangre de todos aquellos que indiquen que su sangre no puede ser usada para transfusión, es removida inmediatamente sin importar los resultados de las pruebas de descarte de enfermedades transmitidas a través de la sangre.

En la década del 80 a raíz de la emergente epidemia de SIDA, se empezó a dar mas importancia a identificar donantes de sangre de alto riesgo, y se empieza a llevar a cabo campañas de difusión a través de los medios de comunicación mas usados (radio, televisión y periódicos) para que aquellos con antecedentes de riesgo se excluyan de participar en el procedimiento. Un estudio en EEUU en 1986 estimó que solo el 61- 67\% de los hombres y $52-55 \%$ de mujeres eran elegibles para donar sangre, considerando solo excluir aquellos con test para hepatitis no-A no-B positivas; pero el número de donantes elegibles continua en descenso conforme nuevas restricciones son añadidas. Tal es la preocupación, que algunos teóricos sugieren cambiar a un sistema de donantes pagados, estrictamente depurados y controlados; o que la institución ofrezca ventajas en el pago de ciertos impuestos para los donantes (1).

En nuestro hospital no se han llevado a cabo trabajos previos, por lo cual decidimos realizar el presente trabajo, cuyo objetivo fue comparar las características sociodemográficas entre donantes de los años 1988 y 1994, con el fin de buscar variaciones que pudieran sugerir la efectividad de aquellas acciones que fomentan la autoexclusión de donantes con antecedente de riesgo.

\section{MATERIAL Y METODOS}

\section{Diseño del estudio}

Se elaboró un estudio retrospectivo comparativo entre grupos históricos.

\section{Población de estudio}

Todos los donantes que acudieron al Banco de Sangre del Hospital Nacional Cayetano Heredia, fueron sometidos a una encuesta después de efectuar su donación. En 1988 se contabilizaron 2148 donantes y en 1994 un total de 2417 donantes, tomándose muestras aleatorias de 627 y 606 donantes respectivamente.

\section{Encuestas}

Todos los donantes fueron sometidos a una encuesta luego de efectuar su donación para identificar aquellos que tenían algún antecedente de riesgo para la transmisión de enfermedades a través de transfusiones.

La encuesta fue realizada por un entrevistador que por lo regular fue el mismo técnico que ejecutaba la extracción.

Las encuestas variaron de formato en el periodo de estudio; desde 1988 hasta 1990 se usó un formato de 40 preguntas y posteriormente uno de sólo 12 preguntas. Los puntos evaluados incluían donaciones previas, parentesco con el receptor, transfusiones recibidas previamente, enfermedades previas (malaria, brucelosis, fiebre tifoidea y hepatitis), número de parejas sexuales en los 6 meses previos, antecedente de relaciones sexuales homosexuales o con prostitutas o personas desconocidas. También extracciones dentales, tatuajes, acupuntura en los 6 meses previos, viaje al 
exterior desde 1980, uso de drogas, permanencia en la cárcel o en el ejército y la motivación para la donación.

En las bolsas de sangre donadas se realizaron las siguientes pruebas serológicas: VDRL-STS, ELISA HbsAg y ELISA VIH.

\section{Análisis estadístico}

La información obtenida se ingresó a una base de datos (Quatro Pro V.6 Borland Int.) a partir de la cual fue procesada estadísticamente mediante el programa Epi-Info V.6 aplicándose las pruebas de Chi-Cuadrado con corrección de Yates y la Prueba Exacta de Fisher.

\section{RESULTADOS}

En 1988 se identificaron 54/2148 (2.5\%) y en 1994 41/2417 (1.7\%) seropositivos para las pruebas mencionadas (Cuadro $\mathrm{N}^{\circ} 1$ ). En ambos años se observó un predominio del sexo masculino (88.3\% en 1988 y $79.5 \%$ en 1994). En el año 1994 hubo más donantes mujeres que en $1988,20.4 \%$ vs $11.6 \%$ respectivamente $(\mathrm{p}<0.0001)$. La mayor proporción de donantes, para ambos años se encontró en el rango de edades entre los 18-25 años, pero en 1994 se observó un incremento en el porcentaje de donantes entre los 36 y 60 años. La edad promedio fue de $30.5 \pm 8.35$ años y $32.5 \pm 9.4$ años en los años 1988 y 1994, respectivamente. (Cuadro $\mathrm{N}^{\mathrm{o}}$ 2).

La mayoría de donantes tenía educación secundaria o superior (86.4\% en 1988 y $88.2 \%$ en 1994). Entre los varones, aquellos con educación secundaria o superior, representaban el 92.2\% (437/474) en 1994 y el 86.4\% (362/419) en 1988 ( $\mathrm{p}<0.01)$. En cuanto al estado civil, los solteros representaron el $47.3 \%$ en 1988 y el

Cuadro $\mathrm{N}^{\circ} 1$. Resultados de los estudios serológicos
por año de estudio.

\begin{tabular}{lcc} 
& 1988 & 1994 \\
& $n=2148$ & $n=2417$ \\
\hline VDRL & $29(1.35 \%)$ & $25(1.03 \%)$ \\
HBsAg & $24(1.12 \%)$ & $15(0.62 \%)$ \\
VIH & $1(0.05 \%)$ & $1(0.04 \%)$ \\
\hline
\end{tabular}

$39.4 \%$ en 1994. En los varones el porcentaje con pareja estable (convivientes y casados) fue significativamente mayor en 1994 que en $1988,59.2 \%$ (282/476) y $50.8 \%$ (224/441) respectivamente ( $\mathrm{p}=0.01)$, lo que no se observó en las mujeres, $58.5 \%$ (72/123) en 1994 y $58.5 \%$ (31/53) en 1988.

El porcentaje de donantes que donaban por primera vez fue de 51.3\% (311/606) en 1994 y 32\% (201/627) en 1988 ( $\mathrm{p}<0.0001)$. Entre estos $17.9 \%(36 / 201)$ en 1988 y $32.5 \%(101 / 311)$ en 1994 fueron mujeres (p<0.001). El 56.3\% (160/284) de donantes de 1988 manifestó realizar la donación para un familiar, en comparación con el 71.1\% (426/599) de 1994 $(\mathrm{p}<0.0001)$. La frecuencia de transfusiones autólogas permanece baja $(0.4 \%$ en 1988 y $0.9 \%$ en 1994). El $0.8 \%$ (5/627) de donantes de 1988 manifestó haber recibido transfusiones en los últimos 6 meses, mientras que en 1994 fue de 3.1\% (19/606).

El 98\% (615/627) de donantes en 1988 manifestó no haber sufrido ninguna de las enfermedades consultadas, lo que disminuyó en 1994, constituyendo el 93.2\% (565/ 606) $(\mathrm{p}<0.0001)$.

El 49.8\% (164/329) de donantes de 1988, manifestó no haber tenido ninguna pareja sexual en los 6 meses previos y el 35.8\% (118/329) dijo haberlo hecho con una sola pareja; mientras que en 1994 fue de 12\% (72/ $599)$ y $79 \%(475 / 599)$ respectivamente. El $2.5 \%$ (16/ 627) de donantes en 1988 manifestó haber experimentado relaciones homosexuales alguna vez en su vida, lo cual contrastó con el 0\% (0/312) en 1994 $(\mathrm{p}<0.01)$. El 8.3\% (52/627) de donantes en 1988 reconoció haber tenido relaciones sexuales con prostitutas o desconocidos en los últimos 6 meses, mientras que en 1994 fue el 6.4\% (20/312), haciendo la salvedad nuevamente de que la pregunta en este caso se extendió a toda la vida. Por último, el 2.2\% (14/627) de donantes en 1988 manifestó haber consumido drogas alguna vez; en 1994 no se incluyó esta pregunta.

\section{DISCUSION}

Es necesario recalcar que la donación en nuestro hospital es sui generis por que se trata de una donación designada, por que se ejecuta a nombre de un probable receptor. Estas donaciones designadas tienen alto componente de presión social por lo que muchas respuestas de la encuesta pueden tener sesgo a pesar de que se tomaron posterior a la donación.

El porcentaje de donantes mujeres se incrementó para el año 1994 llegando a la cifra de $20.4 \%$, pero manteniéndose entre valores descritos en la literatura 
Cuadro №2. Características socio-demográficas del donante de sangre, años 1988 y 1994.

\begin{tabular}{|c|c|c|}
\hline & 1988 & 1994 \\
\hline EDAD promedio & $30.5 \pm 8.3$ & $32.5 \pm 9.4$ \\
\hline $\begin{aligned} & \text { SEXO } \\
&- \text { Hombres/mujeres } \\
&\end{aligned}$ & $554 / 73$ & $482 / 124$ \\
\hline $\begin{array}{rc}\text { GRADO DE INSTRUCCIÓN } \\
-\quad \text { Secundaria o superior } \\
-\quad \text { Primaria o analfabeto } \\
\end{array}$ & $\begin{array}{c}405 / 468(86.5 \%) \\
63 / 468(13.5 \%) \\
\end{array}$ & $\begin{array}{c}525 / 595(88.2 \%) \\
70 / 595(11.8 \%) \\
\end{array}$ \\
\hline $\begin{aligned} & \text { ESTADO CIVIL } \\
&- \text { Casado o conviviente } \\
&- \text { Soltero, viudo o divorciado. } \\
&\end{aligned}$ & $\begin{array}{l}255 / 494(51.6 \%) \\
239 / 494(48.4 \%) \\
\end{array}$ & $\begin{array}{l}354 / 599(59.1 \%) \\
245 / 599(40.9 \%)\end{array}$ \\
\hline 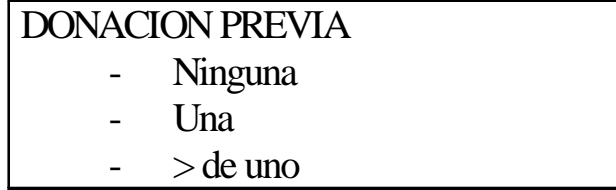 & $\begin{array}{l}201 / 627(32.0 \%) \\
250 / 627(39.9 \%) \\
176 / 627(28.1 \%) \\
\end{array}$ & $\begin{array}{l}311 / 606(51.3 \%) \\
148 / 606(24.4 \%) \\
147 / 606(24.3 \%) \\
\end{array}$ \\
\hline $\begin{aligned} & \text { PARENTESCO CON EL RECEPTOR } \\
& \text { - } \text { Familiar } \\
&- \text { No familiar }\end{aligned}$ & $\begin{array}{l}160 / 285(56.1 \%) \\
124 / 285(43.5 \%)\end{array}$ & $\begin{array}{l}426 / 605(70.4 \%) \\
173 / 605(28.6 \%)\end{array}$ \\
\hline $\begin{aligned} \text { TRANSFUSION PREVIA } \\
-\quad \text { Sí } \\
-\quad \text { No }\end{aligned}$ & $\begin{array}{c}5 / 627(0.8 \%) \\
622 / 627(99.2 \%)\end{array}$ & $\begin{array}{c}19 / 606(3.1 \%) \\
587 / 606(96.6 \%)\end{array}$ \\
\hline $\begin{array}{l}\text { ENFERMEDADES PREVIAS } \\
-\quad \text { Ninguna }\end{array}$ & $615 / 627(98.0 \%)$ & $565 / 606(93.2 \%)$ \\
\hline $\begin{aligned} & N^{\circ} \text { PAREJAS SEXUALES (6m previos) } \\
&- \text { Ninguna } \\
&- 1 \\
&->1 \\
&\end{aligned}$ & $\begin{array}{c}164 / 329(49.8 \%) \\
118 / 329(35.8 \%) \\
7 / 329(14.4 \%) \\
\end{array}$ & $\begin{array}{c}72 / 599(12.0 \%) \\
475 / 599(79.3 \%) \\
52 / 599(8.7 \%)\end{array}$ \\
\hline $\begin{aligned} & \text { CONTACTO HOMOSEXUAL } \\
&-\quad \text { Sí } \\
&- \text { No } \\
&\end{aligned}$ & $\begin{array}{c}16 / 627(2.5 \%) \\
611 / 627(97.5 \%)\end{array}$ & $\begin{array}{c}0 / 312(0.0 \%) \\
312 / 312(100.0 \%)\end{array}$ \\
\hline $\begin{array}{c}\text { CONTACTO CON PROSTITUTAS } \\
-\quad \text { Sí } \\
-\quad \text { No }\end{array}$ & $\begin{array}{c}52 / 627(8.3 \%) \\
575 / 627(91.7 \%)\end{array}$ & $\begin{array}{c}20 / 312(6.4 \%) \\
292 / 312(93.6 \%)\end{array}$ \\
\hline
\end{tabular}


del 66-91\% (2,3). Estudios previos señalan además que entre los que donaban por primera vez, las mujeres eran predominantes con cifras entre $58 \%$ y $66 \%$ (1). En nuestro estudio, si bien la proporción de mujeres que donaban por primera vez aumentó entre 1988 y 1994 de $17.9 \%$ a $32.5 \%$, siempre se observa predominio masculino.

La mayor proporción de donantes, en los dos años evaluados, se encontró en el grupo de 18-25 años, pero la proporción de donantes mayores (>40 años) se incrementó en 1994. Otros estudios revelan edades promedio que oscilan entre 33 y 38 años (1), promedios ligeramente mayores que el nuestro, de 30.5 y 32.5 años en 1988 y 1994 respectivamente. Por otro lado el incremento de donantes de edad mayor no es consistente con las observaciones que realizan otros autores. En los bancos de sangre se estila indicar la edad de 60 años como límite para donar sangre; sin embargo Pindyck señala que las personas de mayor edad son donantes potenciales más confiables por su conducta sexual menos promiscua (4).

El 86.4\% y el $88.2 \%$ en 1988 y 1994,respectivamente, manifestaron tener por lo menos educación secundaria, cifras que son mayores al encontrado en la población general en el Censo de Población y Vivienda del año 1993 para la población mayor de 15 años en la provincia de Lima, de $76.4 \%$ (5). Estudios previos corroboran esta observación, que los donantes de sangre tienen mayor nivel de instrucción que los no-donantes (1) posiblemente por que las personas instruidas tienen mayor chance de leer acerca de las necesidades de sangre y entender lo que eso significa.

La mayoría de los estudios muestran que los donantes son predominantemente casados, en porcentajes que llegan al $73 \%$, superando los niveles de la población general (1). En nuestro estudio los porcentajes de donantes que manifestaron mantener una relación estable (casados y convivientes) fueron $51.7 \%$ en 1988 y $59.1 \%$ en 1994 , cifras mayores que el $47 \%$ encontrado por el Censo de Población y Vivienda de 1993 en la población mayor de 12 años de la provincia de Lima (5).

El 32\% en 1988 y el 51\% en 1994 manifestaron estar donando por primera vez; y aunque la cifra aumentó significativamente en el último año, no significa necesariamente que sea mejor, ya que se ha observado que la frecuencia de marcadores serológicos en este grupo es mayor que en aquellos que han donado previamente, quienes ya han pasado por un screening previo, y saben si deben o no hacer una nueva donación
(51.6\% de nuestros seropositivos donaban por primera vez).

La mayoría de nuestros donantes manifestó que el receptor de su donación era un familiar, tendencia que se incrementa en 1994, esto podría significar una reducción de la donación comercial. Esto en cierto modo es mas seguro ya que no se recurre a personas extrañas, cuyas costumbres se desconoce y más bien se recurre a personas dentro de la familia, que probablemente sean más confiables.

El porcentaje de donantes que se presentó para una transfusión autóloga fue menor del $1 \%$; creemos que debe incentivarse mas este tipo de donación, ya que es la más segura, en los casos en que es factible. Esto no significa que deba pretenderse ofrecerlo como una panacea; en los países desarrollados esta forma de donación alcanza el $10 \%$ del promedio de todas las transfusiones (6).

El porcentaje de donantes que habían recibido transfusiones previas fue mayor en 1994 (3.1\%) que en $1988(0.8 \%)$, pero no podemos hacer comparaciones ya que en 1994 la pregunta incluía toda la vida del donante y no los 6 meses previos a la donación como lo hizo la encuesta de 1988. El hecho de haber recibido una transfusión, por si solo no es tan importante como el mismo antecedente en los 6 meses previos, lo que sería de alto riesgo por el periodo ventana de las enfermedades que hemos comentado anteriormente.

$\mathrm{Al}$ indagar acerca de enfermedades transmisibles, como malaria, brucelosis, fiebre tifoidea o hepatitis, solo el $2 \%$ en 1988 y el $6.8 \%$ en 1994, manifestaron haber padecido de alguna de estas enfermedades. El incremento significativo durante el último año podría explicarse por que en 1988 no se indagó por fiebre tifoidea, antecedente cuya importancia para decidir una transfusión es aún discutible, pudiéndose afirmar que solo un pequeño porcentaje de donantes tenía este antecedente de riesgo.

La gran mayoría refirió haber tenido relaciones sexuales con al menos una pareja sexual y no haberlo hecho con una pareja homosexual. En 1994 se observó una disminución aunque no significativa, de aquellos que manifestaron haber tenido relaciones sexuales con prostitutas o personas desconocidas, factor de riesgo conocido para adquirir enfermedades de transmisión sexual. Se indagó en 1988 por consumo de drogas, dato que fue positivo en el $2.2 \%$ de donantes, este grupo de donantes ha sido señalado como de alto riesgo por 
la elevada promiscuidad y prevalencia de enfermedades de transmisión sexual que presentan $(7,8)$.

En conclusión, hemos encontrado que el porcentaje de antecedentes de riesgo en donantes de sangre de 1994 fue menor que en 1988, con lo cual podría haberse reducido la transmisión de enfermedades infecciosas en el periodo ventana, ya que en cuanto a la serología no hubo diferencia significativa. La adecuada selección de donantes, el uso racional de las transfusiones y el screening serológico constituyen los principales medios para asegurar la inocuidad de la hemoterapia.

\section{Correspondencia:}

De la Cruz Del Solar, Rafael.

Dirección: Jr. Bolognesi 217-K Lima 17. Lima, Perú.

E-mail: rafaelde@medscape.com

\section{BIBLIOGRAFIA}

1. Piliavin J. A. Why do they give the gift of live? A review of research on blood donors since 1977. Transfusion 1990; 30:444-459.

2. Oswalt R.M. A review of blood donor motivation and recruitment. Transfusion 1977; 17:123-135.

3. Burnett J.J. Examining the profiles of the donor and non donor through a multiple discriminant approach. Transfusion 1982; 22:138-142.

4. Pindyck J., Avorn J et al. Blood donation by the elderly. JAMA 1987; 257:1186-8.

5. Instituto Nacional de Estadística e Informática. Censos Nacionales 1993. IX de Población y IV de Vivienda. Perfil Socio Demográfico. INEI Colección Análisis Censal, 1993.

6. Giordano G.F., Dockery J. et al. An autologous blood program coordinated by a regional blood center: a 5 years experience. Transfusion 1991; 31:509-512.

7. Vidal J., Valdez H. Y Adachi R. Prevalencia de prácticas de riesgo y seropositividad para VIH en un grupo de dependientes de TBC en tratamiento. Libro de Resúmenes del V Congreso Panamericano de Infectología. 1991. Lima - Perú.

8. Farfán G., Vidal J. y col. Marcadores serológicos de hepatitis viral B en pacientes drogadictos del Hospital Herminio Valdizán. Rev Gastroenterol Perú 1989; 9:9599. 